\title{
OGLE-LMC-ECL-11893: THE DISCOVERY OF A LONG-PERIOD ECLIPSING BINARY WITH A CIRCUMSTELLAR DISK
}

\author{
Subo Dong ${ }^{1}$, Boaz Katz ${ }^{2,11}$, Jose L. Prieto ${ }^{3,12}$, Andrzej Udalski ${ }^{4,13}$, Szymon Kozlowski ${ }^{4,13}$,

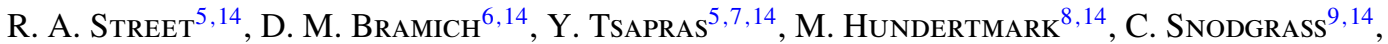 \\ K. Horne ${ }^{8,14}$, M. DominiK ${ }^{8,14,15}$, AND R. Figuera JaImes ${ }^{8,10,14}$ \\ ${ }^{1}$ Kavli Institute for Astronomy and Astrophysics, Peking University, Yi He Yuan Road 5, Hai Dian District, Beijing 100871, China \\ 2 Institute for Advanced Study, 1 Einstein Drive, Princeton, NJ 08544, USA \\ ${ }^{3}$ Department of Astrophysical Sciences, Princeton University, 4 Ivy Lane, Peyton Hall, Princeton, NJ 08544, USA \\ ${ }^{4}$ Warsaw University Observatory, Al. Ujazdowskie 4, 00-478 Warszawa, Poland \\ ${ }^{5}$ Las Cumbres Observatory Global Telescope Network, 6740 Cortona Drive, suite 102, Goleta, CA 93117, USA \\ ${ }^{6}$ Qatar Environment and Energy Research Institute, Qatar Foundation, Tornado Tower, Floor 19, P.O. Box 5825, Doha, Qatar \\ ${ }^{7}$ School of Physics and Astronomy, Queen Mary University of London, Mile End Road, London E1 4NS, UK \\ ${ }^{8}$ SUPA, School of Physics and Astronomy, University of St Andrews, North Haugh, St Andrews KY16 9SS, UK \\ ${ }_{9}^{9}$ Max Planck Institute for Solar System Research, Justus-von-Liebig-Weg 3, D-37077 Göttingen, Germany \\ ${ }^{10}$ European Southern Observatory, Karl-Schwarzschild-Straße 2, D-85748 Garching bei München, Germany \\ Received 2014 March 5; accepted 2014 April 15; published 2014 May 20
}

\begin{abstract}
We report the serendipitous discovery of a disk-eclipse system OGLE-LMC-ECL-11893. The eclipse occurs with a period of 468 days, a duration of about 15 days, and a deep (up to $\Delta m_{I} \approx 1.5$ ), peculiar, and asymmetric profile. A possible origin of such an eclipse profile involves a circumstellar disk. The presence of the disk is confirmed by the $\mathrm{H}-\alpha$ line profile from the follow-up spectroscopic observations, and the star is identified as Be/Ae type. Unlike the previously known disk-eclipse candidates, the eclipses of OGLE-LMC-ECL-11893 retain the same shape throughout the span of $\sim 17 \mathrm{yr}$ (13 orbital periods), indicating no measurable orbital precession of the disk.
\end{abstract}

Key words: binaries: eclipsing - circumstellar matter - stars: individual (OGLE-LMC-ECL-11893) - stars: variables: T Tauri - Herbig Ae/Be

Online-only material: color figures, machine-readable table

\section{IDENTIFICATION OF THE PECULIAR ECLIPSES OF OGLE-LMC-ECL-11893}

The OGLE LMC eclipsing binary (EB) catalog (Graczyk et al. 2011) reported the discovery of 26121 EBs toward the LMC. In 2011 November, while searching the catalog for long-period (period $P>100$ days), highly eccentric binaries (Dong et al. 2013), we serendipitously identified a very unusual EB OGLE-LMC-ECL-11893 (R.A.: 05:17:21.17 decl.: $-69: 00: 55.7$; J2000) by visual inspection. The periodfolded $I$-band light curve shows an eclipse with a peculiar shape (Figure 1). The data are taken from phases II and III of the OGLE survey (Udalski et al. 1997, 2008) and span 12 yr (further data from OGLE-IV survey are discussed in Section 2). The period is 468.124 days, and the baseline I-band magnitude is about 17.6. Each eclipse occurrence is shown with a different color. The eclipse bears some close similarity to EE Cephei, an eclipsing Be star with a circumstellar disk (Gałan et al. 2012), but also has some unique characteristics. In the following, we list several striking features of the OGLE-LMC-ECL-11893 eclipses.

1. The eclipse profile, which spans about 15 days, has an unusual asymmetric shape that cannot be produced by a spherical occulting star. The shape of the profile bears a close similarity to EE Cephei during its 2008/9 eclipse (e.g., see the right panel of Figure 3 in Gałan et al. 2012) and

\footnotetext{
11 John N. Bahcall Fellow.

12 Carnegie-Princeton Fellow.

13 The OGLE Collaboration.

14 The RoboNet Collaboration.

15 Royal Society University Research Fellow.
}

the duration of EE Cephei is also similar ( 230 days). The ingress and egress of OGLE-LMC-ECL-11893 are steeper than those of EE Cephei.

2. The eclipse is deep, with a maximal depth of $\sim 1.5$ mag or equivalent, and $75 \%$ of the $I$-band light is occulted. This is comparable to that of EE Cephei, whose eclipse depths vary between $\sim 0.5$ and 2.0 mag (Gałan et al. 2012).

3. The shape of the eclipse does not change with time. The nine eclipses spanning $17 \mathrm{yr}$ detected so far have exactly the same profile within measurement uncertainties. This is in contrast with EE Cephei and three other well-known disk-eclipse events ( $\varepsilon$ Aurigae, OGLE-LMC-ECL-17782, and $\mathrm{KH} 15 \mathrm{D})$, whose eclipse depths vary from one orbital period to another. These variations are generally attributed to the precessions of the disks.

The similarities between OGLE-LMC-ECL-11893 and EE Cephei hint that they have similar physical origins, possibly disk-eclipsing events, as suggested by Gałan et al. (2012) for EE Cephei.

\section{FOLLOW-UP OBSERVATIONS}

We obtained two optical spectra of OGLE-LMC-ECL-11893, first on 2011 December 23 with IMACS f/2 (Dressler et al. 2011) mounted on the Magellan Baade $6.5 \mathrm{~m}$ and the second on 2013 January 7 with the Magellan Echellette spectrograph (MagE; Marshall et al. 2008). For the IMACS f/2 spectrum, we used the $3001 \mathrm{~mm}^{-1}$ grism and 0.7 slit, which gives resolution $R=1800$ and wavelength coverage 3700-9500 A. We show the IMACS spectrum of the star in the upper panel of Figure 2. MagE with $1^{\prime \prime}$ slit gives $R=4800$ and wavelength 


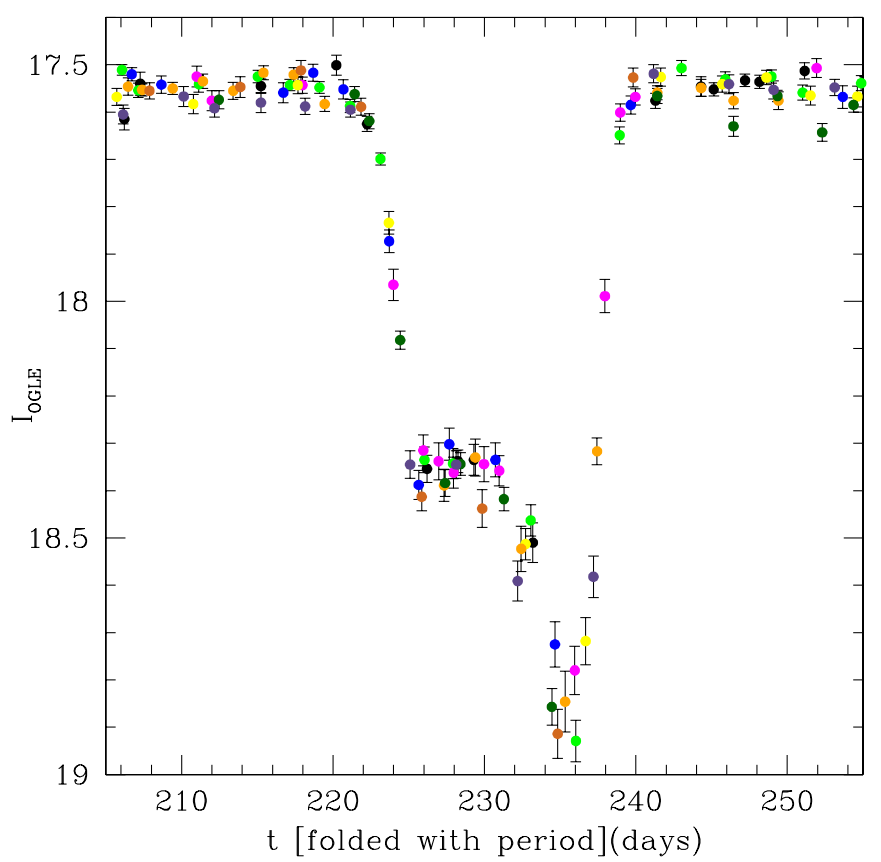

Figure 1. Period-folded OGLE I-band light curve of OGLE-LMC-ECL-11893 with respect to $\mathrm{HJD}_{0}=2,454,245.5663$. Photometry from different orbital periods is shown in different colors. The observations by OGLE-II and OGLE-III span $\sim 12 \mathrm{yr}$ and contain nine eclipse occurrences. The peculiar and asymmetric eclipse profile is unchanged within measurement uncertainties during the span of observations.

(A color version of this figure is available in the online journal.)

coverage 3300-10,000 $\AA$. The IMACS spectrum was reduced with standard techniques in IRAF. The MagE spectrum was reduced with the Carnegie pipeline from D. Kelson.

Using the Ulyss package, which constrains stellar parameters by fitting stellar spectra with the Elodie spectroscopy library (Koleva et al. 2009), we find that the spectrum is consistent with that of a late B/early A-type giant with $T_{\text {eff }} \sim 10,000 \mathrm{~K}$. The RV of the star measured from the MagE spectrum is $270 \mathrm{~km} \mathrm{~s}^{-1}$, consistent with the radial velocity (RV) of the LMC. In both spectra, we find $\mathrm{H}-\alpha$ line profiles characteristic of circumstellar disks (Silaj et al. 2010), confirming the existence of the disk indicated by the eclipse and that the system is of Be/Ae type. The $\mathrm{H}-\alpha$ profile in the MagE spectrum is shown in the lower panel of Figure 2. The $\mathrm{H}-\alpha$ profile is similar to that of $\varepsilon$ Aurigae (Silaj et al. 2010), typical for a Be star observed edge-on (see Figure 3 in Slettebak 1979). $\varepsilon$ Aurigae is a disk-eclipsing event for which the disk was directly confirmed by infrared interferometry (Kloppenborg et al. 2010).

Multi-band photometry obtained using the $1 \mathrm{~m}$ telescopes (Domes A and B) at LCOGT's site in Chile (CTIO) during 2013 February and March (at the early commissioning stages of the telescopes) are shown in Figure 3 and given in Table 1. The observations were conducted through the Observation Control interface developed for the RoboNet project, taken in semirobotic mode as the underlying software was in the process of being upgraded to integrate with the new $1 \mathrm{~m}$ network at the time. The image data were preprocessed using LCOGT's Standard Pipeline, which is based around ORAC-DR, after which the RoboNet pipeline (based on DanDIA by Bramich 2008) was used for difference image analysis photometry.

The different bands (filled and empty dots) are compared to the folded $I$ and $V$ bands OGLE II, III, and IV photometry (empty
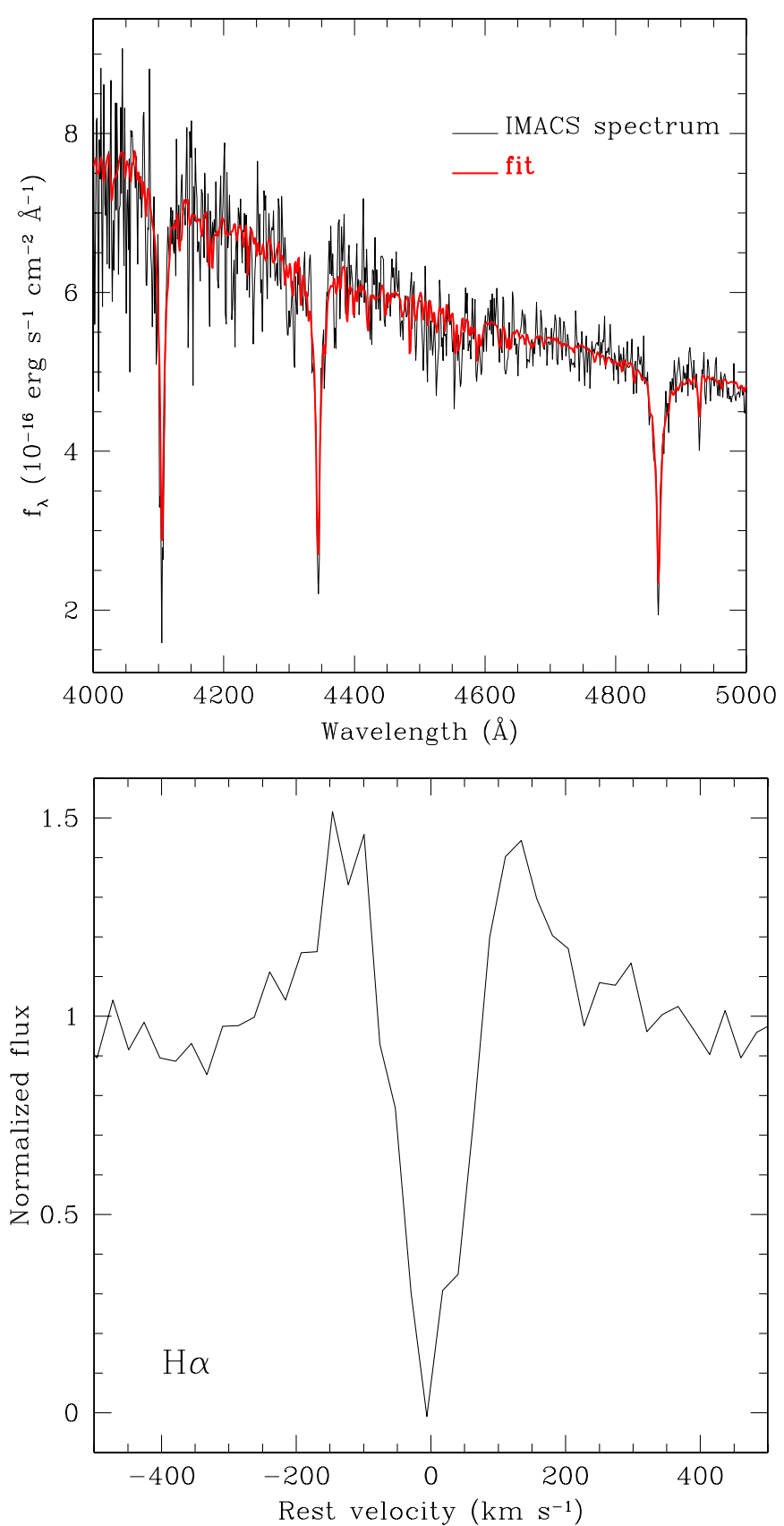

Figure 2. Top: spectrum of OGLE-LMC-ECL-11893 taken by IMACS (black). The best-fit model from Ulyss (red) suggests it is a late B/early A-type giant. Bottom: the $\mathrm{H}-\alpha$ line profile in the MagE spectrum that is characteristic of $\mathrm{Ae} / \mathrm{Be}$ stars confirms the existence of a circumstellar disk (Silaj et al. 2010). The same profile also clearly shows in the IMACS spectrum, though the H- $\alpha$ region is near the edge of the CCD. The H- $\alpha$ profile is typical for an edge-on Be star, similar to that of $\varepsilon$ Aurigae (see bottom left panel of Figure 8 on page 241 in Silaj et al. 2010), for which the disk was directly confirmed by infrared interferometer imaging (Kloppenborg et al. 2010).

(A color version of this figure is available in the online journal.)

squares and filled and empty pentagons). Given the photometric uncertainties in the LCOGT data, there is reasonable agreement between the LCOGT data and the OGLE data for the $V$ and $I$ bands where they could be directly compared. While most bands have a roughly similar profile, the eclipse depth seems to increase for bluer bands (from $I, R, V$, to $B$ ). In particular, the $B$ band seems to have a consistently deeper eclipse and the two $B$-band measurements during HJD 2,456,353, in the deepest point of the eclipse, seem to be particularly deep compared to 
Table 1

Time-series $B, V, R$, and $I$ Photometry for OGLE-LMC-ECL-11893 from the LCOGT Network of Telescopes

\begin{tabular}{|c|c|c|c|c|c|c|c|c|c|}
\hline $\begin{array}{l}\text { Telescope } \\
\text { ID }\end{array}$ & Filter & $\begin{array}{l}\text { HJD } \\
\text { (days) }\end{array}$ & $\begin{array}{l}m_{\text {ins }} \\
(\mathrm{mag})\end{array}$ & $\begin{array}{c}\sigma_{m} \\
(\mathrm{mag})\end{array}$ & $\begin{array}{c}f_{\text {ref }} \\
\left(\mathrm{ADU} \mathrm{s}^{-1}\right)\end{array}$ & $\begin{array}{c}\sigma_{\text {ref }} \\
\left(\mathrm{ADU} \mathrm{s}^{-1}\right)\end{array}$ & $\begin{array}{c}f_{\text {diff }} \\
\left(\mathrm{ADU} \mathrm{s}^{-1}\right)\end{array}$ & $\begin{array}{c}\sigma_{\text {diff }} \\
\left(\mathrm{ADU} \mathrm{s}^{-1}\right)\end{array}$ & $p$ \\
\hline 1sc-doma-1m0-05-kb78 & $B$ & $2,456,343.58641$ & 21.502 & 0.034 & 69.323 & 1.879 & -43.868 & 0.773 & 0.9913 \\
\hline 1sc-doma-1m0-05-kb78 & $B$ & $2,456,343.59010$ & 21.404 & 0.032 & 69.323 & 1.879 & -41.498 & 0.804 & 0.9908 \\
\hline$\vdots$ & $\vdots$ & $\vdots$ & : & : & $\vdots$ & $\vdots$ & $\vdots$ & $\vdots$ & : \\
\hline 1sc-doma- $1 \mathrm{m0} 0-05-\mathrm{kb} 78$ & $V$ & $2,456,343.60545$ & 21.035 & 0.021 & 38.004 & 3.224 & 0.599 & 0.819 & 1.0731 \\
\hline 1sc-doma-1m0-05-kb78 & $V$ & $2,456,343.68000$ & 21.011 & 0.026 & 38.004 & 3.224 & 1.451 & 0.976 & 1.0451 \\
\hline$\vdots$ & $\vdots$ & $\vdots$ & $\vdots$ & $\vdots$ & $\vdots$ & $\vdots$ & $\vdots$ & $\vdots$ & $\vdots$ \\
\hline lsc-domb-1m0-09-kb73 & $I$ & $2,456,364.58433$ & 20.885 & 0.019 & 43.815 & 2.107 & 0.426 & 0.787 & 1.0018 \\
\hline 1sc-domb-1m0-09-kb73 & $I$ & $2,456,364.58801$ & 20.923 & 0.020 & 43.815 & 2.107 & -1.077 & 0.786 & 1.0024 \\
\hline
\end{tabular}

Notes. The instrumental $m_{\text {ins }}$ magnitudes are listed in Column 4 corresponding to the LCOGT site/dome/telescope/instrument code, filter, and epoch of mid-exposure listed in Columns 1-3, respectively. The uncertainty on $m_{\text {ins }}$ is listed in Column 5 . For completeness, we also list the quantities $f_{\text {ref }}, f_{\text {diff }}$, and $p$ in Columns 6, 8, and 10, along with the uncertainties $\sigma_{\text {ref }}$ and $\sigma_{\text {diff }}$ in Columns 7 and 9 . Note that $m_{\text {ins }}(t)=25-2.5 \log \left(f_{\text {ref }}+\left(f_{\text {diff }}(t) / p(t)\right)\right)$, where $t$ is time. Note that these magnitudes are not calibrated to standard systems and they should be used only for studying the relative flux changes. The calibrated magnitudes of the system at the baseline are: $I=17.55 \pm 0.03$, $V=17.74 \pm 0.02$, and $B=17.95 \pm 0.04$.

(This table is available in its entirety in a machine-readable form in the online journal. A portion is shown here for guidance regarding its form and content.)

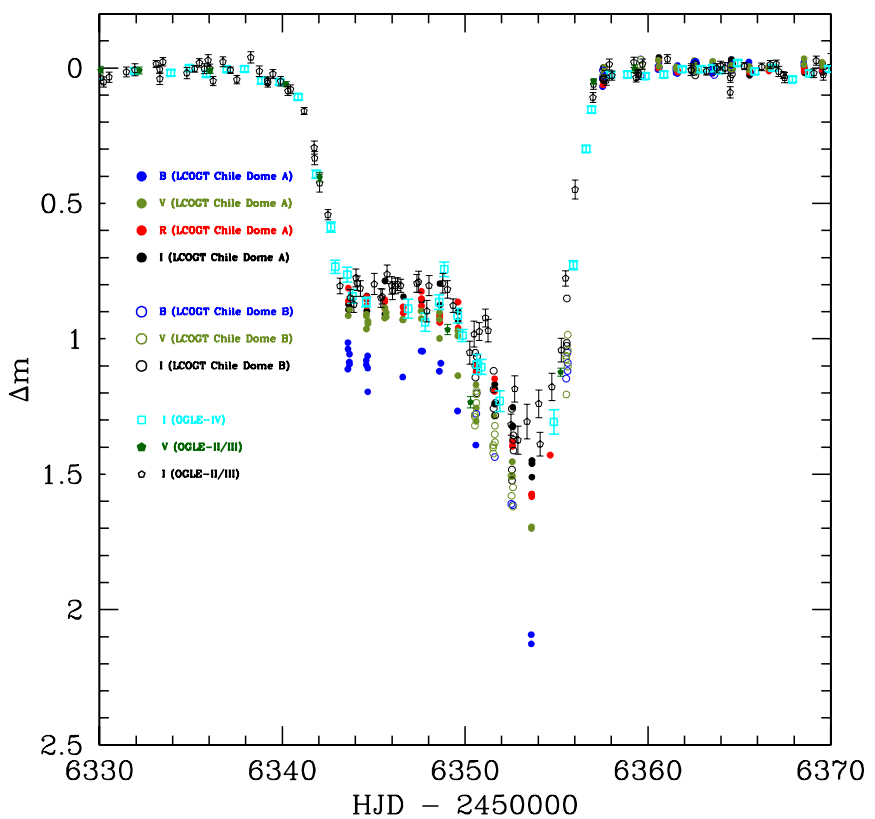

Figure 3. Multi-band follow-up light curves of the OGLE-LMC-ECL-11893 $B V R I$ filters (in blue, olive green, red, black) were taken by LCOGT Chilean $1 \mathrm{~m}$ telescopes (Dome A: filled circles, Dome B: open circles) during the 2013 eclipse. The OGLE-IV I-band observations (cyan open squares) were taken in the 2013 and 2011 seasons. The OGLE-III and OGLE-II I-band (black open pentagons) and $V$-band (dark green filled pentagons) observations are also shown for comparison. Observations which were not taken in 2013 are shifted by periods to match the time of the eclipse in 2013 .

(A color version of this figure is available in the online journal.)

other bands. The color variations during the eclipse events may be related to several physical properties of the system, such as possible temperature variations across the eclipsed areas, the opaqueness of the disk as a function of wavelength, the limb darkening profile of the eclipsed stars, etc. Further highprecision observations are required to accurately measure these differences and study their implications.

The next two eclipses of OGLE-LMC-ECL-11893 will be around HJD 2,456,818 (2014 June) and 2,457,286 (2015 September). However, the former will be practically unobserv- able as it will be too close to the Sun. We encourage intense multi-band photometric and spectroscopic monitoring during the eclipse. In addition, long-term RV monitoring will be useful to determine the orbital parameters and the mass of the eclipsing component.

\section{DISCUSSION}

Assuming a circular orbit, the size of an eclipsing region is of the order of: $D \sim 2 \pi a \Delta t / P=\Delta t\left(2 \pi G M_{\text {tot }} / P\right)^{1 / 3} \sim$ $0.35\left(M_{\mathrm{tot}} / 3 M_{\odot}\right)^{1 / 3} \mathrm{AU}$ for eclipse duration $\Delta t \sim 15$ days and $P=468$ days, where $M_{\mathrm{tot}}$ is the total mass of the system and $a$ is the semi-major axis.

Three well-known periodic disk-eclipse EB candidates are $\varepsilon$ Aurigae $(P=27 \mathrm{yr})$, EE Cephei $(P=5.6 \mathrm{yr})$, and OGLELMC-ECL-17782 ( $P=13.4$ day, identified by Graczyk et al. 2011 in the OGLE LMC EB catalog). The remarkably complicated eclipses of KH 15D are interpreted as a circumbinary disk eclipsing a pair of stars (Winn et al. 2006). Evidences for disk-eclipsing nature of young stellar objects YLW 16A and WL 4 have been presented in Plavchan et al. (2013) and Parks et al. (2014). An additional disk-eclipse candidate with one occurrence of eclipse was suggested by Mamajek et al. (2012). A LMC Be star, FTS ID 78.5979.72, which had a sudden transition from no measurable variabilities to eclipsing with short time variabilities, was reported in Struble et al. (2006).

The period, depth, eclipse duration, and stellar type of OGLELMC-ECL-11893 are similar to those of EE Cephei, suggesting a similar origin for the peculiar eclipses in both systems. The $\mathrm{H}-\alpha$ line profile is similar to that of $\varepsilon$ Aurigae. One important difference is that the shape of OGLE-LMC-ECL-11893 appears to be constant while those of EE Cephei, $\varepsilon$ Aurigae, OGLELMC-ECL-17782, and KH 15D change between eclipses, interpreted as precessions of disks. OGLE-LMC-ECL-11893 is exceptional in this respect in that it does not have measurable precession. Another interesting qualitative difference between OGLE-LMC-ECL-11893 and EE Cephei is that the former has relatively short durations of the ingress and egress than the latter. The disk-eclipse scenario along with other physical properties of the system will be studied in depth by E. Scott et al. (2014, in preparation). 
The serendipitous identification of OGLE-LMC-ECL-11893 demonstrates the power of the OGLE survey in making discoveries of unanticipated variables, thanks to its long-term, highcadence, and high quality photometry of a large number of stars. This has implications for planning future large time-domain surveys such as LSST because the overwhelming majority of such systems in the Galaxy would lie in the inner two quadrants of the Galactic disk, a region that was excluded in the recently published LSST planning (see Figure 1 of Gould 2013 and Figure 4.4 of Abate et al. 2012).

We thank Cullen Blake for stimulating discussion on alternative models and the participants of a lively Institute for Advanced Study astro-coffee session held in 2011 December. We are grateful to Alice Quillen and Josh Winn for helpful comments. S.D. is supported by "the Strategic Priority Research ProgramThe Emergence of Cosmological Structures" of the Chinese Academy of Sciences (grant No. XDB09000000). The OGLE project has received funding from the European Research Council under the European Community's Seventh Framework Programme (FP7/20072013)/ERC grant agreement No. 246678 to A.U. M.D., R.F.J., K.H., R.A.S., C.S., and Y.T. are supported by NPRP grant NPRP-09-476-1-78 from the Qatar National Research Fund (a member of Qatar Foundation). D.M.B.'s contribution to this publication was made possible by NPRP grant \# X-019-1-006 from the Qatar National Research Fund (a member of Qatar Foundation). C.S. has received funding from the European Union Seventh Framework Programme (FP7/2007-2013) under grant agreement No. 268421.

\section{REFERENCES}

Abate, A., Aldering, G., Allen, S. W., et al. 2012, arXiv:1211.0310 Bramich, D. M. 2008, MNRAS, 386, L77

Dong, S., Katz, B., \& Socrates, A. 2013, ApJL, 763, L2

Dressler, A., Bigelow, B., Hare, T., et al. 2011, PASP, 123, 288

Gałan, C., Mikołajewski, M., Tomov, T., et al. 2012, A\&A, 544, A53

Gould, A. 2013, arXiv:1304.3455

Graczyk, D., Soszyński, I., Poleski, R., et al. 2011, AcA, 61, 103

Kloppenborg, B., Stencel, R., Monnier, J. D., et al. 2010, Natur, 464, 870

Koleva, M., Prugniel, P., Bouchard, A., \& Wu, Y. 2009, A\&A, 501, 1269

Mamajek, E. E., Quillen, A. C., Pecaut, M. J., et al. 2012, AJ, 143, 72

Marshall, J. L., Burles, S., Thompson, I. B., et al. 2008, Proc. SPIE, 7014, 701454

Parks, J. R., Plavchan, P., White, R. J., \& Gee, A. H. 2014, ApJS, 211, 3

Plavchan, P., Güth, T., Laohakunakorn, N., \& Parks, J. R. 2013, A\&A, 554, A 110

Silaj, J., Jones, C. E., Tycner, C., Sigut, T. A. A., \& Smith, A. D. 2010, ApJS, 187,228

Slettebak, A. 1979, SSRv, 23, 541

Struble, M. F., Galatola, A., Faccioli, L., Alcock, C., \& Cruz, K. 2006, AJ, 131,2196

Udalski, A., Kubiak, M., \& Szymanski, M. 1997, AcA, 47, 319

Udalski, A., Szymanski, M. K., Soszynski, I., \& Poleski, R. 2008, AcA, 58,69

Winn, J. N., Hamilton, C. M., Herbst, W. J., et al. 2006, ApJ, 644, 510 